\title{
Ground States of the Spin-One-Half Falicov-Kimball Model with Hund's Rule Coupling in Two Dimensions
}

\author{
H. Čenčariková, P. Farkašovský, N. Tomašovičová \\ AND M. ŽONDA \\ Institute of Experimental Physics, Slovak Academy of Sciences \\ Watsonová 47, 04001 Košice, Slovakia
}

\begin{abstract}
The spin-one-half Falicov-Kimball model with spin-dependent on-site interaction between localized $(f)$ and itinerant $(d)$ electrons is studied by small-cluster exact-diagonalization calculations and a well-controlled approximative method in two dimensions. The results obtained are used to categorize the ground-state configurations according to common features (charge and spin ordering) for all $f$ and $d$ electron concentrations $\left(n_{f}\right.$ and $\left.n_{d}\right)$ on finite square lattices. It is shown that only a few configuration types form the basic structure of the charge phase diagram in the $n_{f}-n_{d}$ plane. In particular, the largest regions of stability correspond to the phase segregated configurations and configurations that can be considered as mixtures of chessboard configurations and the full (empty) lattice. Since the magnetic phase diagram is much richer than the charge phase diagram, the magnetic superstructures are examined only at selected values of $f$ and $d$ electron concentrations.
\end{abstract}

PACS numbers: 75.10.Lp, 71.27.+a, 71.28.+d

\section{Introduction}

The interplay between charge and spin degrees of freedom in strongly correlated systems has attracted an enormous interest in recent years due to the rich variety of charge and spin orderings found in some rare-earth and transition-metal compounds. Charge and spin superstructures have been observed, for example, in doped niclate [1], cuprate [2] and cobaltate [3] materials some of which constitute materials that exhibit high-temperature superconductivity. In order to describe both types of ordering in the unified picture, a simple model based on a generalization of the spin-one-half Falicov-Kimball model (FKM) with an anisotropic, spin-dependent interaction that couples the localized and itinerant subsystems was proposed [4]. Thus the model Hamiltonian can be written as

$$
\begin{aligned}
H & =\sum_{i j \sigma} t_{i j} d_{i \sigma}^{+} d_{j \sigma}+U \sum_{i \sigma \sigma^{\prime}} f_{i \sigma}^{+} f_{i \sigma} d_{i \sigma^{\prime}}^{+} d_{i \sigma^{\prime}} \\
& +J \sum_{i \sigma}\left(f_{i-\sigma}^{+} f_{i-\sigma}-f_{i \sigma}^{+} f_{i \sigma}\right) d_{i \sigma}^{+} d_{i \sigma}
\end{aligned}
$$

where $f_{i \sigma}^{+}\left(f_{i \sigma}\right)$ are the creation (annihilation) operators for an electron of spin $\sigma=\uparrow$ and $\downarrow$ in the localized state at lattice site $i$ and $d_{i \sigma}^{+}\left(d_{i \sigma}\right)$ are the creation (annihilation) operators of the itinerant electrons in the $d$-band Wannier state at site $i$. The first term of (1) is the kinetic energy corresponding to quantum-mechanical hopping of the itinerant $d$ electrons between sites $i$ and $j$. These intersite hopping transitions are described by the matrix elements $t_{i j}$, which are $-t$ if $i$ and $j$ are the nearest neighbors and zero otherwise (in the following all parameters are mea- sured in units of $t$ ). The second term represents the on-site Coulomb interaction between the $d$-band electrons with density $n_{d}=N_{d} / L=\frac{1}{L} \sum_{i \sigma} d_{i \sigma}^{+} d_{i \sigma}$ and the localized $f$ electrons with density $n_{f}=N_{f} / L=\frac{1}{L} \sum_{i \sigma} f_{i \sigma}^{+} f_{i \sigma}$, where $L$ is the number of lattice sites. The third term is the above mentioned anisotropic, spin-dependent local interaction of the Ising type between the localized and itinerant electrons that reflects the Hund rule force. Moreover, it is assumed that the on-site Coulomb interaction between $f$ electrons is infinite and so the double occupancy of $f$ orbitals is forbidden. Since the $f$-electron occupation number $f_{i \sigma}^{+} f_{i \sigma}$ of each site $i$ still commutes with the Hamiltonian (1), the $f$-electron occupation number is a good quantum number, taking only two values: $w_{i \sigma}=1$ or 0 , according to whether or not the site $i$ is occupied by the localized $f$ electron. Therefore, the Hamiltonian (1) can be written as $H=\sum_{i j \sigma} h_{i j} d_{i \sigma}^{+} d_{j \sigma}$, where $h_{i j}=t_{i j}+\left(U w_{i}+\right.$ $\left.J w_{i-\sigma}-J w_{i \sigma}\right) \delta_{i j}$. Thus for a given $f$-electron configuration $w=\left\{w_{1}, w_{2}, \ldots, w_{L}\right\}$, the Hamiltonian $H$ is the second-quantized version of the single-particle Hamiltonian $h(w)$, so the investigation of the model is reduced to the investigation of the spectrum of $h$ for different configurations of $f$ electrons. This can be performed exactly, over the full set of $f$-electron distributions (including their spins), or approximatively, over the reduced set of $f$-electron configurations [5]. Here we use a combination of both numerical methods. The main goal for performing these calculations was to construct the comprehensive phase diagram of the spin-1/2 FKM in two dimensions. 
The second goal of our numerical study was to verify the stability of obtained results in the thermodynamic limit by comparing our results with those obtained recently by Lemanski using the method of restricted phase diagrams $[4]$.

\section{Results and discussion}

To construct the comprehensive picture of charge and magnetic ordering in the extended FKM in two dimensions, the complete phase diagram of the model in the $N_{f}-N_{d}$ plane has been calculated point by point for all even numbers of $N_{f}$ and $N_{d}$. Of course, such a procedure demands a considerable amount of CPU time, that imposes severe restrictions on the size of clusters that can be studied numerically $(L=8 \times 8)$. First, we have concerned our attention on the problem of charge ordering. In Fig. 1 we present results of our numerical calculations obtained for $U=8$ and $J=0.5$ in the form of the skeleton phase diagram. One of the most interesting observations is that the phase diagram consists of only a few configuration types, although the total number of possible configurations increases very rapidly with the cluster size $L$ as $3^{L}$. In particular, we have detected 4 different charge configuration types, and namely: (a) the segregated configurations, where $f$ electrons clump together, (b) the $n$-molecular phases, (c) the regular phases and mixtures of regular (usually chessboard) phases and empty/full lattice and (d) the miscellaneous configurations. The typical examples corresponding to these configuration types are depicted on the right side of Fig. 1. As one can see, the largest stability regions correspond to only two configuration types, and namely, the segregated configurations, which fill the left and right side of the phase diagram and the regular phases/mixtures of regular phases and empty (full) lattice localized at the central part of the skeleton phase diagram around the line $N_{f}+N_{d} / 2=L$.

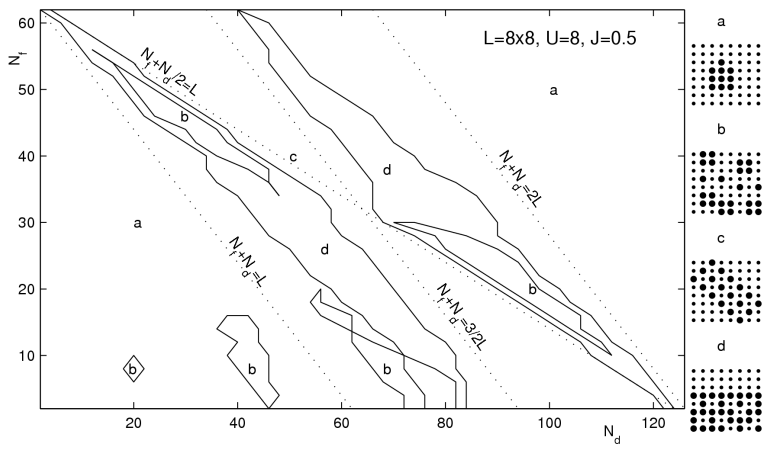

Fig. 1. The skeleton phase diagram of the extended spin-1/2 FKM with some representative configurations. (a) The segregated configurations, (b) the $n$-molecular phases, (c) the regular phases, the mixtures of regular phases and full/empty lattice and (d) the miscellaneous phases. Large dots: sites occupied by $f$ electrons, small dots: vacant sites.

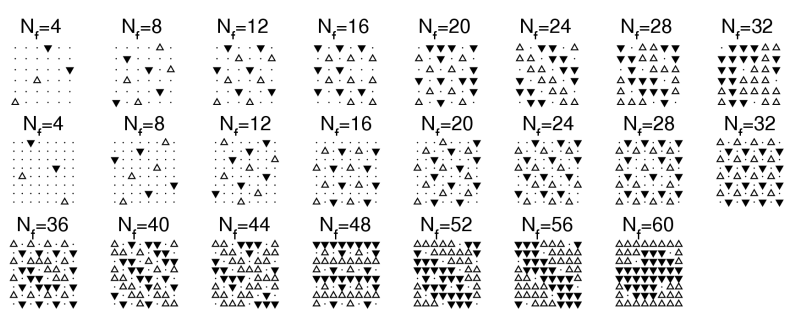

Fig. 2. The ground-state configurations for $N_{f}+$ $N_{d} / 2=L$ calculated by our method on $L=6 \times 6$ and $L=8 \times 8$. To visualize spin distributions we use $\Delta(\boldsymbol{\nabla})$ for the up (down) spin electrons.

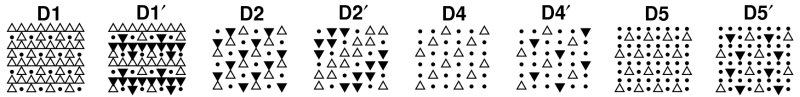

Fig. 3. The ground-state configurations of the spin-1/2 FKM for $U=8$ and four different pairs of $n_{f}$, $n_{d}$ obtained by two different approaches: the method of restricted phase diagrams [4] (D1', D2 ${ }^{\prime}, \mathrm{D} 4^{\prime}$ and $\left.\mathrm{D} 5^{\prime}\right)$ and our numerical method (D1', D2', D4' and D5').

In the next step we perform calculations exclusively at this condition $N_{f}+N_{d} / 2=L$, since for this case there exist numerical results obtained in the thermodynamic limit $(L \rightarrow \infty)$ using the method of restricted phase diagrams [4] that can be used to verify the stability of our solutions. The results of our numerical calculations obtained on clusters of $L=6 \times 6$ and $L=8 \times 8$ sites are summarized in Fig. 2. Analyzing these results we have found that: (i) the system prefers homogeneous distributions of $f$ electrons ( $f$ holes) for $n_{f} \leq 1 / 2\left(n_{f}>1 / 2\right)$; (ii) the ground states are always antiferromagnetic for $n_{f} \leq 1 / 2$ and antiferro-, ferrimagnetic for $n_{f}>1 / 2$; (iii) for $n_{f}>1 / 2$ an obvious tendency to form the striped spin phases is observed. A comparison of our results with the results obtained by the method of restricted phase diagrams (for $n_{f}=3 / 4$ (D1), $2 / 3$ (D2), 1/2 (D3), 1/3 (D4) and $1 / 4$ (D5)) shows (see Fig. 3) that both approaches yield the same results for the charge distributions of $f$ electrons, but differ in a prediction of spin ordering in all cases except the half-filled band case $\left(n_{f}=1 / 2\right)$. To check the stability of our solutions we have performed numerical calculations also for $L=12 \times 12,16 \times 16$ and $18 \times 18$ clusters. The results obtained are shown in Fig. 4 and they clearly demonstrate that ground states found for $L=8 \times 8$ cluster hold also on much higher clusters. This fact allows us to extrapolate our numerical results to the thermodynamic limit, where they can be directly compared with the Lemanski results. This comparison shows (see Fig. 5) that our method yields in all cases the lower energy in the thermodynamic limit $(L \rightarrow \infty)$ than the Lemanski approach. Moreover, a comparison of our and Lemanski's ground states (Fig. 3) provides a direct explanation of this discrepancy, and namely, that the unit cells used in Ref. [4] are too small to describe cor- 


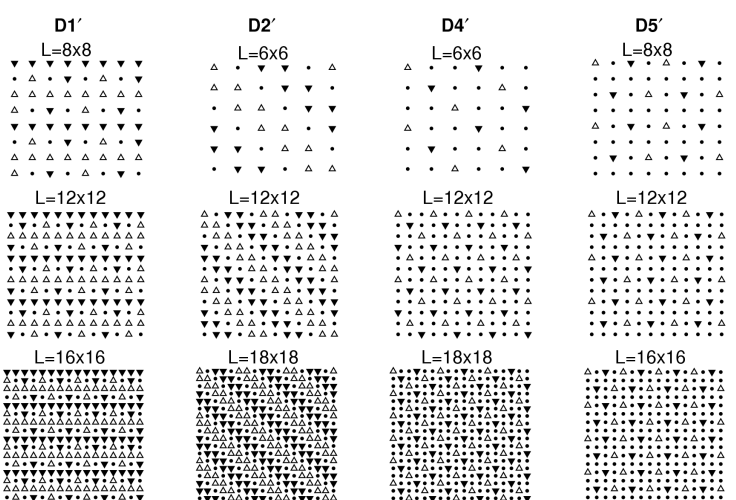

Fig. 4. The ground-state configurations of the two-dimensional spin-1/2 FKM calculated for the same $U, n_{f}$ and $n_{d}$ values as in Fig. 3 on different clusters.

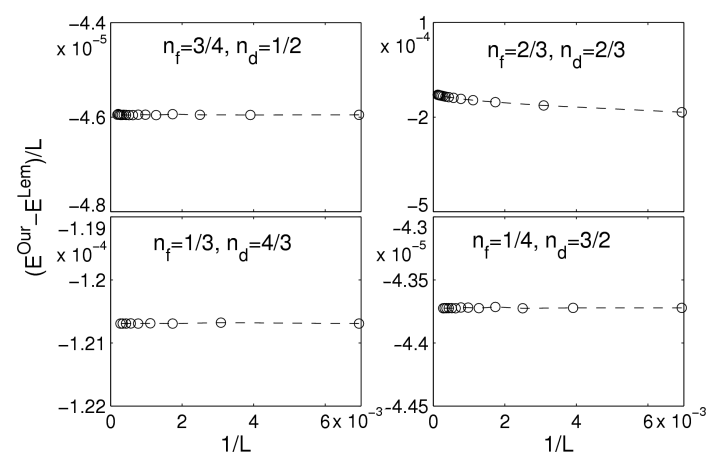

Fig. 5. The difference between our and the Lemanski ground-state energies as a function of $1 / L$ calculated for the extrapolated configurations corresponding to $\mathrm{D} 1^{\prime}$, $\mathrm{D} 2^{\prime}, \mathrm{D} 4^{\prime}, \mathrm{D} 5^{\prime}$ and D1, D2, D4, D5 phases. The lines are only guides to the eye. rectly the spin distributions. This fact leads, of course, to a different magnetic phase diagram in comparison to one presented in Ref. [4], where only antiferromagnetic phases (for $n_{d} \approx 1$ ) and ferromagnetic phases (for $n_{d}$ far away from 1) have been found. In contrast to Ref. [4] we have not observed the stable ferromagnetic phases at $N_{f}+N_{d} / 2=L\left(N_{f} \neq L\right)$ but only antiferromagnetic phases and a few ferrimagnetic phases.

\section{Acknowledgments}

This work was supported by Slovak Grant Agency VEGA under grant No. 2/7057/27 and Slovak Research and Development Agency (APVV) under grant LPP0047-06. H.C. acknowledges support of Stefan Schwartz Foundation.

\section{References}

[1] C.H. Chen, S.W. Cheong, A.S. Cooper, Phys. Rev. Lett. 71, 2461 (1993).

[2] J.M. Tranquada, B.J. Sternlieb, J.D. Axe, Y. Nakamura, S. Uchida, Nature (London) 375, 561 (1995).

[3] K. Takada, H. Sakurai, E. Takayama-Muromachi, F. Izumi, Nature (London) 422, 53 (2003).

[4] R. Lemanski, Phys. Rev. B 71, 035107 (2005).

[5] P. Farkašovský, Eur. Phys. J. B 20, 209 (2001). 